\title{
Astrometric mass ratios for three spectroscopic binaries (Research Note)
}

\author{
J. Sahlmann ${ }^{1}$ and F. C. Fekel ${ }^{2}$ \\ 1 Observatoire de Genève, Université de Genève, 51 chemin Des Maillettes, 1290 Versoix, Switzerland \\ e-mail: johannes.sahlmann@unige.ch \\ 2 Center of Excellence in Information Systems, Tennessee State University, 3500 John A. Merritt Boulevard, Box 9501, Nashville, \\ TN 37209, USA \\ e-mail: fekel@evans.tsuniv.edu
}

Received 13 June 2013 / Accepted 10 July 2013

\section{ABSTRACT}

\begin{abstract}
The orbits of five single-lined spectroscopic binaries have recently been determined. We now use astrometric measurements that were collected with the HiPPARcos satellite to constrain the systems' mass ratios and secondary masses. The barycentric astrometric orbits of three binary systems, HD 140667, HD 158222, and HD 217924, are fully determined and precise estimates of their mass ratios are obtained. Follow-up of these systems with infrared spectroscopy could yield model-independent dynamical masses for all components.
\end{abstract}

Key words. binaries: spectroscopic - stars: low-mass - astrometry - stars: individual: HD 140667 - stars: individual: HD 158222 stars: individual: HD 217924

\section{Introduction}

Spectroscopic binaries make it possible to study stellar multiplicity over a wide range of secondary masses and are therefore one of the foundations for our understanding of star and binary formation (Duquennoy \& Mayor 1991; Raghavan et al. 2010). The orbital elements of a single-lined spectroscopic binary (SB1) produce the mass function $f(m)$, whose value depends on the primary mass $M_{1}$, the secondary mass $M_{2}$, and the inclination of the orbital plane $i$ relative to the plane of the sky, which usually is unknown. If an estimate of $M_{1}$ can be made, the minimum mass of the secondary, $M_{2} \sin i$, is obtained. Therefore, only a lower limit to the secondary-to-primary mass ratio $q=M_{2} / M_{1}$ is known for most SB1s. Astrometric measurements of orbital motion can determine the inclination, and thus the mass ratio. Obtaining a large number of systems with well-determined $q$ values helps to refine our knowledge of the binary population (Tohline 2002; Goodwin 2013) and SB1s with astrometric orbits (e.g. Pourbaix \& Jorissen 2000; Jancart et al. 2005; Ren \& Fu 2013) can complement the samples of doublelined spectroscopic binaries (SB2, e.g. Mazeh et al. 2003) and eclipsing binaries. Here, we study five new SB1s analysed by Fekel et al. (2013) that have primary spectral types ranging from F9 V to G5 V and whose basic properties are given in Table 1. These binaries have eccentricities $e \approx 0.2-0.84$, orbital periods $P \approx 60-2400$ days, and radial velocity (RV) amplitudes of $K_{1} \approx 5-27 \mathrm{~km} \mathrm{~s}^{-1}$.

\section{Primary mass estimates}

Estimates of the primary masses were obtained by determining the effective temperature and luminosity of each star and then comparing those results with theoretical evolutionary tracks. For each system we began by adopting the $V$ mag and $B-V$ colour
Table 1. Primary masses and minimum secondary masses of the five systems.

\begin{tabular}{lccccc}
\hline \hline HD & $\begin{array}{c}f(m) \\
\left(M_{\odot}\right)\end{array}$ & $\begin{array}{c}M_{1} \\
\left(M_{\odot}\right)\end{array}$ & $\begin{array}{c}M_{2} \sin i \\
\left(M_{\odot}\right)\end{array}$ & $\begin{array}{c}P \\
(\text { day })\end{array}$ & $e$ \\
\hline 100167 & 0.0489 & 1.01 & 0.48 & 60.6 & 0.68 \\
135991 & 0.0045 & 1.07 & 0.19 & 151.0 & 0.57 \\
140667 & 0.0100 & 1.04 & 0.26 & 978.4 & 0.20 \\
158222 & 0.0214 & 0.94 & 0.32 & 206.1 & 0.41 \\
217924 & 0.0119 & 1.05 & 0.28 & 2402.7 & 0.84 \\
\hline
\end{tabular}

Notes. Mass function, period, and eccentricity are given for orientation, see exact values in Fekel et al. (2013).

from the Hipparcos catalogue (Perryman et al. 1997). We next adopted the parallax from our revised astrometric solution, if available (see Sect. 3), or the parallax reduction by van Leeuwen (2007). The apparent magnitude and parallax result in the absolute magnitude. The $B-V$ colour in conjunction with Table 3 of Flower (1996) provides the bolometric correction and effective temperature for each star. Converting the bolometric magnitude to luminosity in solar units, we then plotted the stars in a theoretical H-R diagram (Fig. 1) and compared them with the solar abundance evolutionary tracks of Girardi et al. (2000). The primary mass estimates are listed in Table 1 with the corresponding minimum secondary masses.

\section{Analysis of the HIPPARcos astrometry}

All stars listed in Table 1 were catalogued by the HipParcos astrometry satellite (Perryman et al. 1997). We used the new Hipparcos reduction (van Leeuwen 2007) to search for signatures of orbital motion in the Intermediate Astrometric Data 


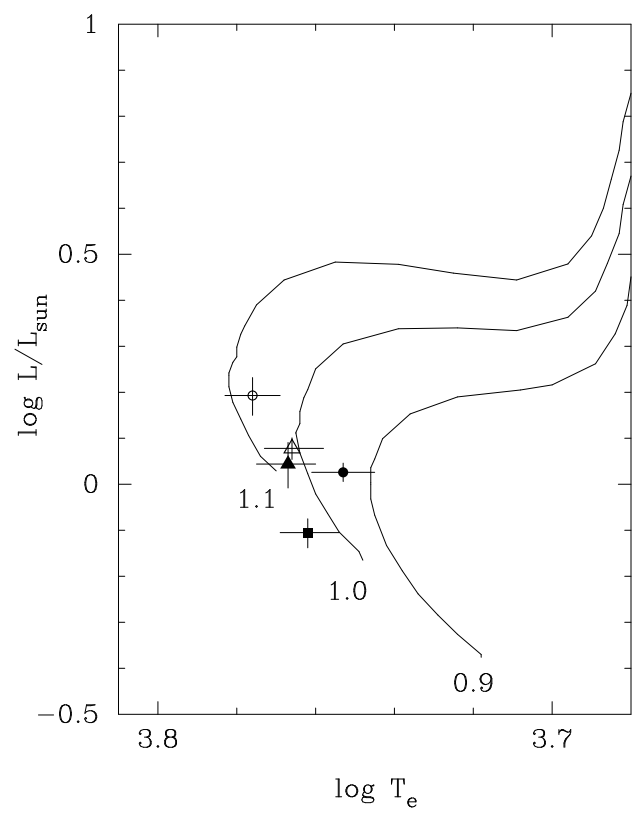

Fig. 1. Hertzsprung-Russell diagram showing the locations of HD 100167 (open triangle), HD 135991 (open circle), HD 140667 (filled triangle), HD 158222 (filled square), and HD 217924 (filled circle). Theoretical evolutionary tracks of solar composition are labelled for masses of $0.9,1.0$, and $1.1 M_{\odot}$.

(IAD). The analysis was performed as described in Sahlmann et al. (2011b), where a detailed description of the method can be found. The main reduction elements are as follows. Using the spectroscopic orbital parameters of Fekel et al. (2013), the IAD was fitted with a seven-parameter model, where the free parameters are the inclination $i$, the longitude of the ascending node $\Omega$, the parallax $\varpi$, and offsets to the coordinates $\left(\Delta \alpha^{\star}, \Delta \delta\right)$ and proper motions $\left(\Delta \mu_{\alpha^{\star}}, \Delta \mu_{\delta}\right)$. A two-dimensional grid in $i$ and $\Omega$ (see Fig. 2) was searched for its global $\chi^{2}$-minimum with a standard nonlinear minimisation procedure. The statistical significance of the derived astrometric orbit was determined with a permutation test employing 1000 pseudo orbits. Uncertainties in the solution parameters were derived by Monte Carlo simulations that include propagation of RV parameter uncertainties. This method has proven to be reliable in detecting orbital signatures in the HIPPARcos IAD (Sahlmann et al. 2011b,a; Díaz et al. 2012). Because the binaries are SB1s, it is reasonable to assume that the secondaries' light has a negligible effect on the HiPPARcos astrometry, i.e. the photocentric and barycentric positions coincide. Table 2 gives the new Hipparcos catalogue parameters of the five primaries. HD 217924 and HD 140667 have a stochastic ("1") and accelerated ("7") solution type, respectively, which is common for systems with unrecognised orbital motion (Sahlmann et al. 2011b). The remaining three systems have standard five-parameter solutions ("5"). The parameter $N_{\text {orb }}$ represents the number of orbital periods covered by the Hipparcos observation timespan and $N_{\text {Hip }}$ is the number of IAD measurements in van Leeuwen (2007) subtracted by the number of measurements $N_{\text {rej }}$ that we rejected. The median astrometric precision is given by $\sigma_{\Lambda}$.

\section{Results}

We performed the analysis for the five systems and found that the orbits of HD 140667, HD 158222, and HD 217924 are constrained by the Hipparcos astrometry. Figures 2 and 3 show the
Table 2. Characteristics of the new reduction HIPPARcos IAD.

\begin{tabular}{lcccccc}
\hline \hline HD & HIP & $\begin{array}{c}\text { Sol. } \\
\text { type }\end{array}$ & $N_{\text {orb }}$ & $\begin{array}{c}\sigma_{\Lambda} \\
\text { (mas) }\end{array}$ & $N_{\text {Hip }}$ & $N_{\text {rej }}$ \\
\hline 100167 & 056257 & 5 & 17.5 & 2.7 & 96 & 0 \\
135991 & 074821 & 5 & 6.5 & 4.3 & 124 & 1 \\
140667 & 077098 & 7 & 0.8 & 2.8 & 48 & 4 \\
158222 & 085244 & 5 & 5.6 & 3.5 & 102 & 1 \\
217924 & 113884 & 1 & 0.5 & 5.2 & 48 & 0 \\
\hline
\end{tabular}

corresponding confidence contours and the barycentric orbits, respectively. Tables 3 and 4 summarise the numerical results. The orbital motion of HD 100167 and HD 135991 is not detected. The systems are discussed individually below.

\subsection{HD 217924}

The astrometric orbit is clearly detected with a significance of $>99.9 \%(>3.3 \sigma)$, which is also reflected in the small residuals of the 7-parameter model $\left(\mathrm{O}-\mathrm{C}_{7}\right)$ including the orbit compared to the standard 5-parameter model residuals $\left(\mathrm{O}-\mathrm{C}_{5}\right)$ and the corresponding small F-test null probability of the simpler model being true. The orbital signature is large with a barycentric semimajor axis of $a_{1}=38.1 \pm 1.8$ mas. As a consequence, the orbital fit results in a significantly different proper motion in right ascension. The orbital inclination is $51.3_{-4.0}^{+4.6 \circ}$ and results in a secondary mass of $0.37 \pm 0.03 M_{\odot}$. The system's relative separation is $a_{\text {rel }} \simeq 146$ mas and may be resolved by future high-resolution observations (e.g. Mason et al. 2001).

\section{2. $H D 158222$}

The astrometric orbit is found with a significance of $99.3 \%$ $(2.7 \sigma)$, which is at the limit of a genuine detection. However, inspection of the confidence contours (Fig. 2) shows that the inclination and $\Omega$ are well-constrained, and so the orbital solution is valid. The orbital inclination is $62.4_{-5.1}^{+5.6 \circ}$ and produces a secondary mass of $0.38 \pm 0.02 M_{\odot}$.

\section{3. $H D 140667$}

The permutation test yields a significance of only $12 \%$ for the orbit of HD 140667, which results in a non-detection of orbital motion on the basis of this criterion. However, the F-test probability is lower than for HD 158222 indicating that orbital motion is present and is manifested in significantly reduced residuals. Inspection of the confidence contours (Fig. 2) and of the orbit (Fig. 3) also strongly support a significant detection. We therefore adopt the formal solution of our analysis and claim that the orbit is constrained by HIPPARcos astrometry. The orbital inclination is then $113.3_{-65}^{+6.0 \circ}$ and produces a secondary mass of $0.29 \pm 0.02 M_{\odot}$. By including the orbital model, the parallax becomes significantly smaller by $\sim 2 \sigma$, thus the distance to the system is now larger. The revised parallax for HD 140667 increases its luminosity $L / L_{\odot}$ from 0.874 to 1.107 and radius $R / R_{\odot}$ from 0.91 to 1.03 , thereby shifting its position in the H-R diagram (Fig. 1) towards better agreement with evolutionary models.

We did not find an explanation for the failure of the permutation test, but we note that it is the only case that we have encountered so far in the analysis of more than 100 systems with identical methods, of which many are reported in Sahlmann et al. (2011a,b), Díaz et al. (2012), and Marmier et al. (2013). For HD 140667, we discarded the four IAD entries taken at satellite 
J. Sahlmann and F. C. Fekel: Astrometric mass ratios for three spectroscopic binaries $(R N)$
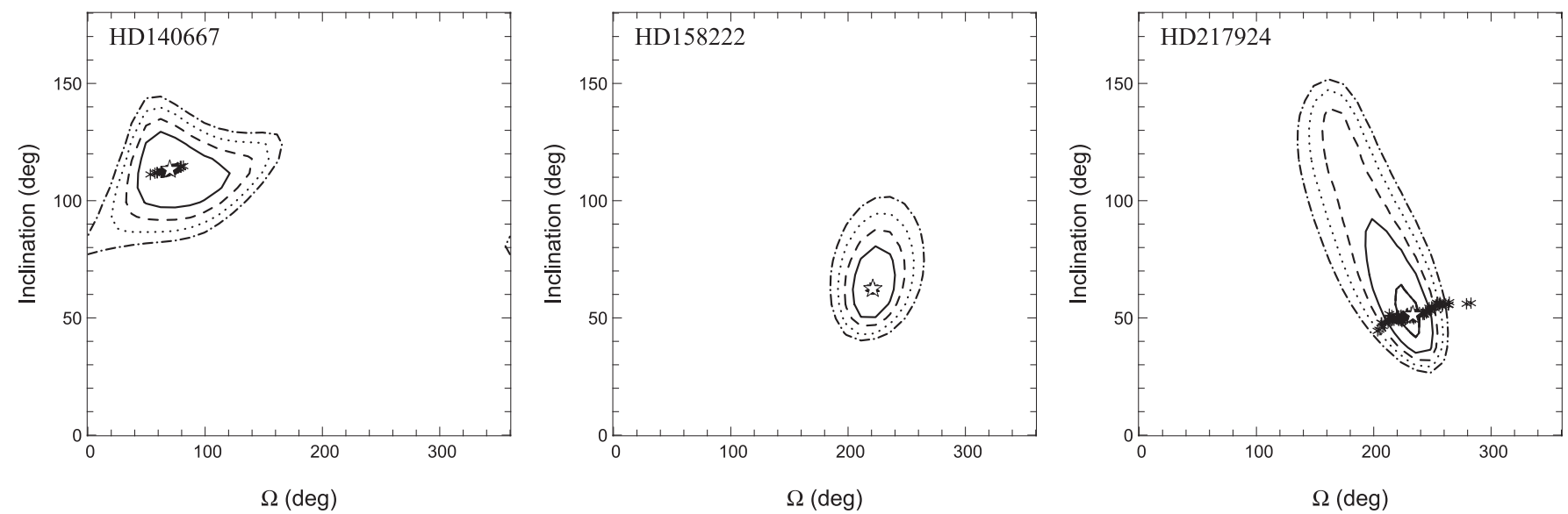

Fig. 2. Joint confidence contours on the $i$ - $\Omega$-grid for HD 140667 (left), HD 158222 (centre), HD 217924 (right). The contour lines correspond to confidences at $1-\sigma$ (solid), 2- $\sigma$ (dashed), 3- $\sigma$ (dotted), and 4- $\sigma$ (dash-dotted) level. The crosses indicate the position of the best non-linear adjustment solution for each of the 100 Monte Carlo samples of spectroscopic parameters and the star corresponds to the adopted orbit.
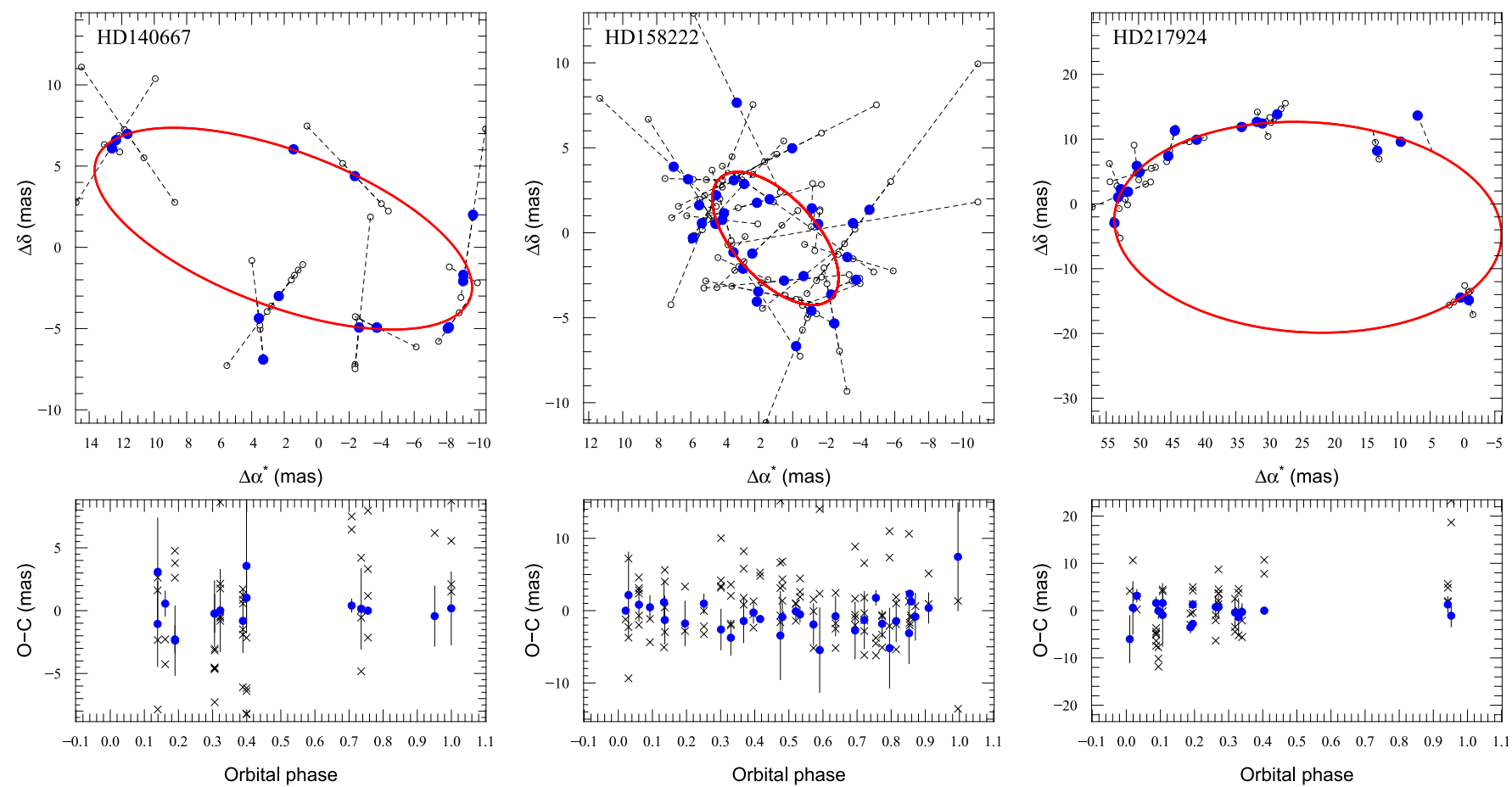

Fig. 3. Visualisation of the barycentric orbits. Top panels: the sky-projected orbits of HD 140667 (left), HD 158222 (centre), HD 217924 (right) are oriented clockwise, counter-clockwise, and counter-clockwise, respectively. North is up and east is left. The solid red line shows the orbital solution and open circles mark the individual HIPPARcos measurements. Bottom panels: the corresponding O-C residuals for the normal points of the orbital solution (filled blue circles) and of the standard 5-parameter model without companion (black crosses).

orbit number 1354, because those had uncertainties $>2 \sigma_{\Lambda}$ and produced abnormally large residuals to the orbital fit (following Sahlmann et al. 2011b). The relatively small number of 48 effectively used IAD measurements is the same as that used for HD 217924 and cannot explain the failure of the permutation test.

\subsection{Companion mass limits for HD 100167 and HD 135991}

Even if evidence of orbital motion is not detected in the astrometric data, we can use the HIPparcos observations to set an upper limit to the companion mass by determining the minimum detectable astrometric signal $a_{\min }$ of the individual target. When the data cover at least one complete orbit,
Sahlmann et al. (2011b,a) have shown that an astrometric signalto-noise of $S / N \gtrsim 6-7$ is required to obtain a detection at the $3 \sigma$ level, where $S / N=a\left(\sigma_{\Lambda} / \sqrt{N_{\text {Hip }}}\right)^{-1}$ and $a$ is the semi-major axis of the detected orbit. Using a conservative $\mathrm{S} / \mathrm{N}$-limit of 8 , we derive the upper companion mass limit $M_{2 \text {,up-lim }}$ as the companion mass which introduces the astrometric signal

$a_{\min }=8 \frac{\sigma_{\Lambda}}{\sqrt{N_{\text {Hip }}}}\left(1-e^{2}\right)$,

where the factor $1-e^{2}$ accounts for the most unfavourable case of $i=90^{\circ}$ and $\Omega=90^{\circ}$ in which the astrometric signal is given by the semi-minor axis of the orbit. This criterion sets mass upper limits of $0.69 M_{\odot}$ and $0.59 M_{\odot}$ to the companions of HD 100167 and HD 135991, respectively. 
Table 3. Astrometric solution parameters for the three significant orbits

\begin{tabular}{lcccccccc}
\hline \hline $\mathrm{HD}$ & $\begin{array}{c}\Delta \alpha^{\star} \\
(\mathrm{mas})\end{array}$ & $\begin{array}{c}\Delta \delta \\
(\mathrm{mas})\end{array}$ & $\begin{array}{c}\varpi \\
(\mathrm{mas})\end{array}$ & $\begin{array}{c}\Delta \varpi \\
(\mathrm{mas})\end{array}$ & $\begin{array}{c}\Delta \mu_{\alpha^{\star}} \\
\left(\mathrm{mas} \mathrm{yr}^{-1}\right)\end{array}$ & $\begin{array}{c}\Delta \mu_{\delta} \\
\left(\mathrm{mas} \mathrm{yr}^{-1}\right)\end{array}$ & $\begin{array}{c}i \\
(\mathrm{deg})\end{array}$ & $\begin{array}{c}\Omega \\
(\mathrm{deg})\end{array}$ \\
\hline 140667 & $-2.7_{-0.9}^{+0.9}$ & $-2.9_{-0.7}^{+0.7}$ & $27.0_{-1.5}^{+1.4}$ & -3.4 & $-7.5_{-2.2}^{+2.2}$ & $-3.5_{-3.5}^{+3.2}$ & $113.3_{-6.5}^{+6.0}$ & $69.9_{-14.7}^{+13.8}$ \\
158222 & $-1.6_{-0.5}^{+0.5}$ & $0.3_{-0.5}^{+0.5}$ & $24.5_{-0.5}^{+0.5}$ & 0.5 & $-1.9_{-0.5}^{+0.5}$ & $-1.5_{-0.6}^{+0.6}$ & $62.4_{-5.1}^{+5.6}$ & $221.1_{-6.8}^{+6.8}$ \\
217924 & $-32.2_{-3.7}^{+3.8}$ & $-4.3_{-9.2}^{+9.8}$ & $37.2_{-1.2}^{+1.3}$ & -1.2 & $-17.0_{-1.7}^{+1.7}$ & $0.2_{-3.8}^{+3.8}$ & $51.3_{-4.0}^{+4.6}$ & $233.4_{-16.5}^{+18.2}$ \\
\hline
\end{tabular}

Table 4. Astrometric solution parameters.

\begin{tabular}{lcccccccccc}
\hline \hline HD & $\begin{array}{c}a \sin i \\
(\mathrm{mas})\end{array}$ & $\begin{array}{c}a \\
(\mathrm{mas})\end{array}$ & $\begin{array}{c}M_{2} \\
\left(M_{\odot}\right)\end{array}$ & $\begin{array}{c}M_{2}(3-\sigma) \\
\left(M_{\odot}\right)\end{array}$ & $\begin{array}{c}a_{\text {rel }} \\
(\mathrm{mas})\end{array}$ & $\begin{array}{c}\mathrm{O}-\mathrm{C}_{5} \\
(\mathrm{mas})\end{array}$ & $\begin{array}{c}\mathrm{O}-\mathrm{C}_{7} \\
(\mathrm{mas})\end{array}$ & $\begin{array}{c}\chi_{7, \text { red }}^{2} \\
(\%)\end{array}$ & $\begin{array}{c}\text { Null prob. } \\
(\%)\end{array}$ & $\begin{array}{c}\text { Significance } \\
(\%)\end{array}$ \\
\hline 100167 & 3.12 & $\ldots$ & $\ldots$ & $\ldots$ & $\ldots$ & 3.52 & 3.40 & 1.35 & 1.4 & 12.9 \\
135991 & 1.80 & $\ldots$ & $\ldots$ & $\ldots$ & $\ldots$ & 5.53 & 5.47 & 1.32 & 9.4 & 6.2 \\
140667 & 12.64 & $12.3_{-0.4}^{+0.4}$ & $0.29_{-0.02}^{+0.02}$ & $(0.26,0.36)$ & 57 & 4.57 & 2.88 & 1.06 & $5.6 \mathrm{e}-08$ & 12.0 \\
158222 & 4.56 & $5.3_{-0.3}^{+0.3}$ & $0.38_{-0.02}^{+0.02}$ & $(0.33,0.46)$ & 18 & 4.58 & 4.04 & 0.93 & $1.0 \mathrm{e}-07$ & 99.3 \\
217924 & 30.72 & $38.1_{-1.8}^{+1.8}$ & $0.37_{-0.03}^{+0.03}$ & $(0.31,0.48)$ & 146 & 6.61 & 2.71 & 0.23 & $1.6 \mathrm{e}-17$ & $>99.9$ \\
\hline
\end{tabular}

Notes. Some parameters are omitted for the two systems with non-detected orbits.

Table 5. Mass ratio constraints.

\begin{tabular}{|c|c|c|}
\hline Primary & $\begin{array}{c}M_{1} \\
\left(M_{\odot}\right)\end{array}$ & $q$ \\
\hline HD 100167 & $1.01[ \pm 0.10]$ & $0.48-0.68$ \\
\hline HD 135991 & $1.07[ \pm 0.11]$ & $0.18-0.55$ \\
\hline HD 140667 & $1.04[ \pm 0.10]$ & $0.27_{-0.02}^{+0.01}\left[\begin{array}{l}+0.03 \\
-0.02\end{array}\right]$ \\
\hline HD 158222 & $0.94[ \pm 0.09]$ & $0.40_{-0.02}^{+0.02}\left[\begin{array}{c}+0.04 \\
-0.04\end{array}\right]$ \\
\hline HD 217924 & $1.05[ \pm 0.11]$ & $0.35_{-0.03}^{+0.03}\left[\begin{array}{l}+0.04 \\
-0.03\end{array}\right]$ \\
\hline
\end{tabular}

Notes. Uncertainties in brackets originate in the systematic primary mass uncertainty of $10 \%$.

\section{Discussion}

The resulting mass ratios for the five SB1s are listed in Table 5. We adopted a systematic $10 \%$ model uncertainty for the primary mass estimate, which is propagated to the mass ratio uncertainty and is larger than the formal uncertainty obtained from the astrometric orbit fitting. For the two systems without detected orbits, we give the acceptable range in mass ratio. In Fig. 4, we compare our results with the sample of 32 SB2s with determined mass ratio from Mazeh et al. (2003). Although the SB1 primaries are on average $\sim 1.4$ times more massive than the primaries of the SB2s examined by Mazeh et al. (2003), their mass ratios appear to be consistent with those of the lower mass primaries. If the SB1s studied here can be converted to SB2s using infrared spectroscopy, the detection of the astrometric orbit will yield modelindependent dynamical masses for all components.

Acknowledgements. J.S. thanks the Swiss National Science Foundation for supporting this research and kindly acknowledges support as a visitor at the Centro de Astrobiología in Villanueva de la Cañada (Madrid). The work of F.C.F. is partially supported by the state of Tennessee through its Centers of Excellence program.

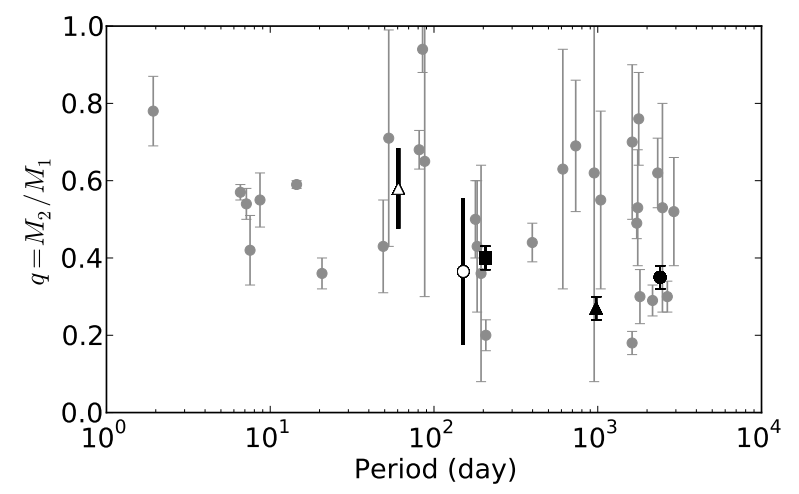

Fig. 4. Mass ratios as a function of period for the five SB1s are shown in black (symbols as in Fig. 1) and the SB2 mass ratios from Mazeh et al. (2003) are shown in grey.

\section{References}

Díaz, R. F., Santerne, A., Sahlmann, J., et al. 2012, A\&A, 538, A113 Duquennoy, A., \& Mayor, M. 1991, A\&A, 248, 485

van Leeuwen, F. 2007, Hipparcos, the New Reduction of the Raw Data, Astrophysics and Space Science Library (Berlin: Springer), 350

Fekel, F. C., Rajabi, S., Muterspaugh, M. W., \& Williamson, M. H. 2013, AJ, 145,111

Flower, P. J. 1996, ApJ, 469, 355

Girardi, L., Bressan, A., Bertelli, G., \& Chiosi, C. 2000, A\&AS, 141, 371

Goodwin, S. P. 2013, MNRAS, 430, L6

Jancart, S., Jorissen, A., Babusiaux, C., \& Pourbaix, D. 2005, A\&A, 442, 365

Marmier, M., Ségransan, D., Udry, S., et al. 2013, A\&A, 551, A90

Mason, B. D., Hartkopf, W. I., Holdenried, E. R., \& Rafferty, T. J. 2001, AJ, 121, 3224

Mazeh, T., Simon, M., Prato, L., Markus, B., \& Zucker, S. 2003, ApJ, 599, 1344 Perryman, M. A. C., Lindegren, L., Kovalevsky, J., et al. 1997, A\&A, 323, L49 Pourbaix, D., \& Jorissen, A. 2000, A\&AS, 145, 161

Raghavan, D., McAlister, H. A., Henry, T. J., et al. 2010, ApJS, 190, 1

Ren, S., \& Fu, Y. 2013, AJ, 145, 81

Sahlmann, J., Lovis, C., Queloz, D., \& Ségransan, D. 2011a, A\&A, 528, L8

Sahlmann, J., Ségransan, D., Queloz, D., et al. 2011b, A\&A, 525, A95

Tohline, J. E. 2002, ARA\&A, 40, 349 